\title{
Emotion Regulation in Adolescents: An ERP study.
}

\section{Authors:}

Alex Desatnik, Tarik Bel-Bahar, Tobias Nolte, Michael Crowley, Peter Fonagy \& Pasco Fearon

\section{Affiliations:}

Alex Desatnik, Pasco Fearon, Peter Fonagy - Research Department of Clinical, Educational and Health Psychology, University College London \& The Anna Freud National Centre for Children and Families. London, United Kingdom.

Tarik Bel-Bahar - Center for Consciousness Science, Department of Anesthesiology, University of Michigan Medical School, Ann Arbor, Michigan, USA

Tobias Nolte - Wellcome Trust Centre for Neuroimaging, University College London, London,United Kingdom

Michael Crowley - Child Study Center, Yale University, New Haven, USA

Correspondence concerning this article should be addressed to Alex Desatnik, Research Department of Clinical, Educational and Health Psychology, University College London, Gower Street, London WC1E 6BT, United Kingdom. E-mail: alex.des@mail.com

Acknowledgments: This study was funded by the NPSA foundation research grant. 
- $\quad$ Expressive suppression modulated the LPP in adolescents.

- Age-related LPP changes suggest increased expressive suppression efficiency with development.

- A more occipitally-focused LPP in adolescence compared to adult findings.

- The LPP can be an effective tool to study emotion regulation in healthy adolescents. 


\section{Keywords:}

Adolescence

Late positive potential (LPP)

Event-related potentials (ERP)

Emotion

Adolescent brain

Emotion regulation

Response modulation

Expressive suppression 


\begin{abstract}
Aims: The use of emotion regulation strategies can reduce the intensity of negative emotional experiences. Event related potentials (ERPs), specifically the late positive potential (LPP), are known to be sensitive to this modulation in adults. This is the first study to explore the neural correlates of expressive suppression in adolescents. We sought to replicate previous findings from emotion regulation studies with adult populations, show that the LPP can be modulated by expressive suppression in healthy adolescents, and examine the influence of age on LPP changes. Method: ERPs of 53 healthy adolescents (12 to 17 years old) performing an emotion regulation task (expressive suppression) were recorded. Results: Expressive suppression altered the LPP in adolescents with both increases and decreases noted depending on time window and recording site. The LPP during expressive suppression was decreased with increasing age. Conclusions: The findings suggest that 1) the LPP is an effective tool to study processes associated with emotion regulation in adolescents, and 2) expressive suppression, in terms of its neural indicators, seems to become more effective with age. The nature and utility of expressive suppression as a specific form of emotion regulation in adolescents are discussed.
\end{abstract}




\section{Introduction}

The ability to regulate one's emotions is considered essential for mental health (Berking, Wirtz, Svaldi \& Hofmann, 2014; Sheppes, Suri \& Gross, 2015; Tortella-Feliu, Balle \& Sesé, 2010; Van Rheenen \& Rossell, 2014). Difficulties in emotion regulation are a key feature characterizing multiple psychopathologies across the lifespan (Aldao, Nolen-Hoeksema \& Schweizer, 2010; American Psychiatric Association, 2013; Fernandez, Jazaieri \& Gross, 2016; Sheppes, Suri \& Gross, 2015). Emotion regulation is thought to involve interactions between multiple cognitive-affective-behavioral processes and is widely researched in adults (e.g., Butler \& Randall, 2013; Campbell-Sills \& Barlow, 2007) and children (e.g., Adrian, Zeman \& Veits, 2011; Gresham \& Gullone, 2012; Kim-Spoon, Cicchetti \& Rogosch, 2013; Lewis, Lamm, Segalowitz, Stieben \& Zelazo, 2006; Zeman, Cassano, Perry-Parrish, \& Stegall, 2006). Adolescence is a developmental phase which presents with increased demands to regulate one's emotions and behavior during a time characterised by multiple neurophysiological, psychological, and social changes (Casey, 2015; Fuhrmann, Knoll \& Blakemore, 2015; Shulman, Harden, Chein \& Steinberg, 2014). Despite this, only a relatively small number of studies, using mainly self-report methodologies, have explored emotion regulation during adolescence (Gresham \& Gullone, 2012; Penela, Walker, Degnan, Fox \& Henderson, 2015; Silvers et al., 2012; Zimmerman \& Iwanski, 2014), and the associations of emotion regulation with psychopathology (Esbjørn, Bender, Reinholdt-Dunne, Munck \& Ollendick, 2012; Garnefski, Kraaij \& van Etten., 2005; Silk, Steinberg \& Morris, 2003). Thus, the empirical study of emotion regulation processes in adolescence requires continued development.

\section{Emotion Generation and Regulation Strategies}

In the process model of emotion regulation (Gross, 2015; Sheppes et al., 2015), emotional experience and expressions arise as an individual attends to and interprets the current situation in terms of relevance to his goals. The generation of emotion and emotion regulation 
is considered to be complex dynamic processes that unfold over time. While emotion generation is considered to occur mainly in the earlier stages of this model, the emotion regulation strategies subsequently chosen can give rise to a new cycle of emotion experiences and later regulation attempts, thus resulting in continuous multiple interactions across stages. These processes are impacted by the way other people react and the general situation. Overall the processes described appear to include a wide variety of emotion regulation dynamics which differ based on time, interpersonal context and environmental feedback.

Five stages of emotional regulation processes are suggested by Gross: situation selection, situation modification, attentional deployment, cognitive change (e.g reappraisal), and response modulation (e.g suppression). Each of these processes is thought to differ in relation to the point in time at which they can be deployed and in the primary impact they have on the emotion generation process. The first four are considered to be antecedent focused and influence the emotion-generation cycle before emotions are fully expressed. Response modulation is considered to be focused on behavioral expressions, occurring once earlier processes such as situation selection and attentional deployment have been deployed (e.g., covering or hiding already generated emotions). As suggested by Gross and Feldman-Barrett (2011), there are various theoretical perspectives on emotion (e.g., basic, appraisal, social construction) that all suggest that emotion-related episodes unfold over time. However, Gross and Feldman-Barrett note that the same emotion-related process can be considered generative or regulative depending on whether they occur towards the beginning or end of an emotional episode.

There is no a priori assumption about a particular form of emotion regulation being "good" or "bad" (Thompson, Lewis \& Calkins, 2008). Some researchers have tended to make the distinction between cognitive change (reappraisal) as an adaptive response, and response modulation (suppression) as having negative physiological and interpersonal consequences 
(Gross, 1998a, 1998b; Sheppes et al., 2015). Other recent studies tend to show that whether an emotion regulation strategy, such as expressive suppression, is effective or adaptive is context dependent (Paul, Simon, Kniesche, Kathmann \& Endrass, 2013; Zimmerman \& Iwanski, 2014; English et al., 2016). One might say that an excessive reliance on any one emotion regulation strategy, or disregarding the situational demands and context, may lead to adverse consequences. For example, persistent or chronic situation selection, such as avoiding difficult emotionally arousing social situations may give rise to social anxiety. On the other hand, attentional deployment may in some circumstances lead one to miss important situational and social information (Campbell-Sills \& Barlow, 2007; Werner \& Gross, 2010).

\section{Neural Correlates of Emotion Generation}

While the neuroscientific investigation of emotion often uses functional magnetic resonance imaging (fMRI), the use of the electroencephalogram (EEG) is also essential to understand brain dynamics associated with emotion generation and regulation. Averaged trials of EEG time locked to stimulus events (e.g., images, sounds, words) are known as event related potentials (ERPs), which reflect event-related synchronous activity of neuronal ensembles recorded by scalp electrodes. EEG allows for millisecond-level resolution, which is crucial for studying the fast temporal brain dynamics of emotion generation and regulation (Hajcak, MacNamara \& Olvet, 2010). Many ERP studies looking at emotional processing and regulation have focused on the late positive potential (LPP), which is a midline ERP observable around $300 \mathrm{~ms}$ after stimulus onset and which lasts for at least several hundred milliseconds (Hajcak et al., 2010). The LPP has been shown to increase significantly in response to emotional images compared to neutral images (including scenes and faces with neutral content, Kujawa, Klein \& Hajcak, 2012). However, some studies show that the LPP may be driven primarily by the arousal content of emotional images, as the LPP was not sensitive to the specific valence (positive vs negative) of the presented images (e.g., Cuthbert, Schupp, Bradley, Birbaumer \& 
Lang, 2000; Keil, Moratti \& Stolarova, 2003). The modulation of the LPP by emotional stimuli is most visible in occipital, parietal, and central EEG channels (Hajcak et al., 2010). It is thought that LPP enhancement observed when emotional stimuli are presented may reflect the downstream effects of early amygdala activation in the visual occipital cortex (de Rover et al., 2012).

\section{Neural Correlates of Emotion Regulation}

In addition to being sensitive to the emotional content of stimuli in general, it is suggested that the time course of the LPP is an index of emotion regulation processes. Several studies have shown LPP sensitivity to various emotion regulation strategies such as reappraisal (Foti \& Hajcak, 2008; Hajcak \& Nieuwenhuis, 2006; Paul et al., 2013; Thiruchselvam, Blechert, Sheppes, Rydstrom \& Gross, 2011), distraction (Paul, Kathmann \& Riesel, 2016; Thiruchselvam et al., 2011; Uusberg, Thiruchselvam \& Gross, 2014), and suppression (Moser, Hajcak, Bukay \& Simons, 2006; Paul et al., 2013). Distraction (Paul et al., 2013; Thiruchselvam et al., 2011), expressive suppression (Paul et al., 2013) and cognitive suppression (Moser et al., 2006) seem to influence the earlier time windows of the LPP starting around $300 \mathrm{~ms}$. Other studies have shown that cognitive reappraisal can influence both late (Paul et al., 2013; Thiruchselvam et al., 2011), and earlier time windows (Hajcak \& Nieuwenhuis, 2006; Krompinger, Moser \& Simons, 2008; Wessing, Rehbein, Postert, Fürniss \& Junghöfer, 2013). It has been suggested that the earlier positivity recorded in these studies is possibly associated with attending to the stimulus, while the later positivity reflects the cognitive-semantic elaboration of the stimulus (Schupp, Flaisch, Stockburger \& Junghöfer, 2006).

\section{Neural Correlates of Emotion Generation and Regulation in Children and Adolescents}

Childhood and adolescence involve a range of normative brain and psychological changes in terms of emotional experience, regulation, and behavior (Casey, 2015; Fuhrmann 
et al., 2015; Stephanou et al., 2016; Vink, Derk, Hoogendam, Hillegers \& Kahn, 2014). Several brain imaging (e.g., Kadosh et al., 2016; McRae et al., 2012; Perlman \& Pelphrey, 2011; Vijayakumar et al., 2014) and behavioral studies (e.g., Brenning, Soenens, Van Petegem \& Vansteenkiste, 2015; Tottenham, Hare \& Casey, 2011; Zimmerman \& Iwanski, 2014) suggest that emotion regulation improves with brain development. The linear improvement with age between adolescents and young adults in emotion regulation was associated with the differential activity in regions closely associated with reappraisal in adults, including greater left ventrolateral prefrontal cortex (PFC) activity, and decreased temporal-occipital and amygdala activity (McRae et al., 2012; Silvers et al., 2016; Stephanou et al., 2016). Further, there is evidence that emotion regulation becomes more selective and effective with age, as adults adapt the way in which they manage emotions based on experience and the demands of the situation (Carstensen, Fung \& Charles, 2003).

Although there are a large number of brain imaging studies with children using emotional stimuli such as faces and other images, only a small number of studies have focused on the LPP in children, and fewer still on emotion regulation tasks. Hajcak and Dennis (2009) used age appropriate images from the International Affective Picture System (IAPS; Lang, Bradley \& Cuthbert, 2008) and found that, similarly to adults, children (5-8 years old) produced increased LPP in response to emotional images relative to neutral ones. However, in this age group LPP activity seemed more focused at occipital regions as opposed to more centrallyfocused LPPs found in adults. Dennis and Hajcak (2009) also demonstrated that emotion regulation strategies modulated the LPP in children (5 to 10-year-olds) with the LPP being significantly lower following neutral compared to negative interpretations of the images. This was only the case for the middle LPP time window (600 to $1000 \mathrm{~ms}$ ), suggesting that, cognitive reappraisal in children has an impact relatively late in the emotion generation process, although other studies have shown reappraisal effects in early LPP time windows with both adults (e.g. 
Hajcak \& Nieuwenhuis, 2006; Krompinger et al., 2008) and children (Wessing et al., 2015). In contrast to the findings of Dennis and Hajcak (2009), DeCicco and colleagues (2012) showed that while the LPP was indeed larger in response to unpleasant versus neutral images in children (5 to 7 year olds) the LPP was not sensitive to reappraisal. The authors explained this discrepancy with previous findings by suggesting that the use of reappraisal is still developing in younger children and that they are less able to use this strategy to regulate their emotions. This discrepancy also highlighted the importance of ensuring that the sometimes complex instructions involved in regulation tasks (e.g. reappraisal, distraction, etc.) are presented in an age-appropriate way so that the absence of evidence for emotion regulation modulation is not simply a result of a child participant's failure to understand or enact the task requirements. Overall, previous child studies have mostly used cognitively demanding and complex regulation tasks. There is also a lack of child studies examining relatively simpler strategies such as simple distraction or expressive suppression.

The number of LPP studies of emotion processing in adolescence is limited in comparison to child and adult studies. Zhang and colleagues (2012) demonstrated that in adolescents (11 to 17 years old) the LPP is modulated by emotional images from the Chinese affective picture system, with emotional images eliciting larger LPP than neutral ones, and hence showing results similar to findings with adults. In another study, Zhang and colleagues (2013) found that individual differences in harm avoidance in adolescents (11 to 19 years old) predicted LPP amplitudes for positive and negative images, but not for neutral ones. Another study found that adolescent criminal offenders (13 to 17 years old), showed decreased LPP for negative images; however, the non-offenders showed larger LPP amplitudes in response to unpleasant compared to both neutral and positive stimuli while no such emotional LPP modulation observed in the offender group (Pincham, Bryce \& Fearon, 2015). Further, higher LPP during passive viewing of emotional stimuli has been associated with psychiatric 
symptoms in adolescence (Kujawa et al., 2015; Nelson, Perlman, Hajcak, Klein \& Kotov, 2015) and there is evidence of LPP test-retest stability (Kujawa et al., 2013) in children and adolescents.

While developmental research suggests that emotion regulation is undergoing significant development during adolescence (Zimmerman \& Iwanski, 2014), little is known about the neural and behavioral correlates of various forms of emotion regulation in adolescents. To date only one LPP study examined emotion regulation in adolescents. Zhang and colleagues (2014) examined LPP modulation with a distraction condition and reported that LPP amplitudes to negative pictures, relative to positive pictures, were reduced during distraction. Counting backwards by three reduced LPP to only negative pictures in younger adolescents, but it reduced LPP for both positive and negative pictures in the older adolescents. However, the chosen distraction strategy was relatively complex.

It is therefore of importance to further explore the effects of various emotion regulation strategies in adolescents, especially strategies that may be more easily deployed and less cognitively demanding. Using such strategies (i.e., expressive suppression) may have the benefits of simple regulation tasks and unambiguous instructions, thus helping to reduce experimental bias associated with cognitive effort. Several adult studies had investigated the effects of expressive suppression (Cutuli, 2014). While in Gross's model (Sheppes et al., 2015) expressive suppression is thought of as a regulatory strategy deployed after an emotion has been generated, other studies suggest that expressive suppression might also have an effect earlier on in the emotion generation cycle. Specifically Paul and colleagues (2013), showed that expressive suppression modulated the LPP from the very beginning of the LPP. They suggested that expressive suppression instructions may be used as a preparatory regulation tool 
to prevent the elicitation of an emotional response before the onset of stimuli and regardless of its content.

Yet no studies to date have specifically examined expressive suppression effects on the LPP in an adolescent sample. Expressive suppression is an important regulation strategy that has received relatively little research attention even though it may involve brain networks that are different from those activated by reappraisal strategies (Morawetz, Bode, Derntl \& Heekeren, 2016). It is a strategy that may be used in situations when it is necessary to conceal either positive or negative expressions, often in social contexts (English et al., 2016). Much like reappraisal, expressive suppression is likely to be influenced by developmental maturation (Gullone et al., 2016). It is also possible that due to the significant importance of peer relationships in adolescence (Collins \& Laursen, 2004; Giordano, 2003) expressive suppression is a particularly relevant emotion regulation strategy. Overall, expressive suppression is a unique subject of study for a better understanding of both adaptive and maladaptive emotion regulation strategies, and whether they become easier with maturation.

\section{Aims of the Present Study}

While developmental research suggests that emotion regulation is undergoing significant development during adolescence (Gullone et al., 2010; Zimmerman \& Iwanski, 2014), little is known about the neural and behavioral correlates of various forms of emotion regulation in adolescents. The current study had three aims. First, we expected to replicate previous findings from adult studies by showing that emotion regulation (namely expressive suppression) reduces LPP amplitude in adolescents. Second, we expected to show that increasing age is linked to less effortful emotion regulation, as reflected by decreased LPP amplitudes in older participants. Third, due to topographic differences found in the LPP between children and adults we explored LPP variability during emotion regulation in this age group by examining 
differences across occipital, parietal and central-parietal midline channels where LPP was previously reported in child and adult populations (Hajcak \& Dennis 2009; Hajcak \& Nieuwenhuis, 2006).

Due to the relatively broad age range (12-17) of participants included in this study, the expressive suppression protocol used was as simple as possible, benefiting from clear instructions that could easily be followed by an adolescents of any age, minimizing the impact of potential confounds related to cognitive load. This protocol has been used in adult populations and shown to be effective in attenuating the LPP at the earlier time windows, similarly to other "pre-emptive" strategies (Paul et al., 2013).

\section{Method}

\section{Participants}

Participants were fifty-three 12 to 17 year old adolescents $(M=14.43$ years, $S D=1.74)$; 29 females and 24 males. There were seventeen 12 to 13 year olds, twenty 14 to 15 year olds, and sixteen 16 to 17 year olds. The participants were recruited from a diverse community in North West London and all were fluent in speaking and reading in English for at least five years. All were right handed with no chronic illnesses, normal or corrected to normal vision, and no self-reported or parent-reported history of drug or alcohol dependency or diagnosed psychopathology. The participants were paid $£ 20$ as renumeration for their participation in the experiment.

\section{Ethical Considerations}

The parents of all participants who were younger than 16 years old and adolescents aged 16 and older were required to sign an informed consent form that detailed the study rationale and all the procedures. Parents completed the screening questions for participants under 16 
years of age. Participants over sixteen completed the screening questionnaires themselves. Both the parents and the participants were clearly informed that they may withdraw from any part of the study at any point. The study has been granted ethical approval by UCL Research Ethics Committee (ID Number: 1908/001).

\section{Stimuli}

The stimuli were presented on a black background of a 15 inch computer monitor using Eprime 2.0 software. 60 unpleasant and 30 neutral developmentally appropriate images were selected from the IAPS pool (Lang et al., 2008). The $9 \times 7 \mathrm{~cm}$ images were presented at the centre of the screen at a $65 \mathrm{~cm}$ viewing distance. Each image covered the horizontal visual angle of $7.9^{\circ}$ and vertical visual angle of $6.1^{\circ}$. We resized the images so that they were centred on the screen, so as to make it easier for participants to fixate on the image without scanning the full screen and thus preventing larger eye movements.

\section{Procedure}

After obtaining consent from the participants, an EEG net was applied and the participants were given detailed instructions. The experiment consisted of two blocks: in the first block (passive viewing) the participants were instructed to view 30 unpleasant (negative view condition) and 30 neutral (neutral view condition) images presented in random order. This was followed by the expressive suppression condition. In the instructions, participants were asked not to show their feelings, so that any person watching them would have no idea what they were feeling. The participants were shown cameras at the bottom of the computer screen and informed that the experimenters would be watching their responses. Videos were not recorded and used only for 1) monitoring attentiveness in participants and 2) to provide the manner by which the participant would be "watched for signs of emotion" during the expressive suppression task. They were then asked to tell the experimenter what was required of them during the task.Then participants were presented with the expressive suppression block 
consisting of 30 negative images. For all conditions, participants completed three practice trials which they were allowed to repeat if they wished.

The present study did not counterbalance the passive viewing and expressive suppression conditions. This is in line with previous studies investigating the effects of expressive suppression that did not use counterbalancing of conditions (Gross \& Levenson, 1993; Murata, Moser \& Kitayama, 2012; Musser et al., 2011). Conditions were presented in a fixed non-counterbalanced order to minimize potential carry-over effects (Murata et al., 2012; Musser et al., 2011) between conditions, i.e., to avoid participants continuing to suppress in the passive view condition after having done the suppression condition first. Other influences that may have been minimized with the current design include task-switching or cognitive load effects between conditions (Murata et al., 2012; Musser et al., 2011). Further, possible benefits from doing passive viewing first include familiarizing participants with the general requirements of the image-viewing task (Gross \& Levenson, 1993; Musser et al., 2011) and maximizing the potential effects of the suppression challenge (Musser et al., 2011).

Following the completion of the task, the participants were shown 30 random positive images from the IAPS in order to improve their mood in case it was affected by the images they had seen; this last block was not recorded. After the last block the EEG net was removed and the participants were allowed to rest. During that time, the participants were asked what they were doing in the task and all the participants confirmed that they had tried to make sure the experimenters could not see what they felt from their face and (upper) body. Finally the participants were paid and debriefed.

\section{The Task}

Each trial in the passive viewing block began with a white fixation cross that appeared at the centre of the screen for $500 \mathrm{~ms}$, which was followed by a $500 \mathrm{~ms}$ blank screen, after 
which the neutral or unpleasant image was presented for the duration of $2000 \mathrm{~ms}$, which was then followed by another $500 \mathrm{~ms}$ blank screen.

Each trial in the expressive suppression block began with a white fixation cross appearing at the centre of the screen for $500 \mathrm{~ms}$. The cross was followed by a $2000 \mathrm{~ms}$ ("regulate") window during which the words "don't show" appeared on the screen in white on a black background accompanied by a male or female voice (alternating) saying "don't show". This was followed by the presentation of the unpleasant image for $2000 \mathrm{~ms}$ which was followed by a $500 \mathrm{~ms}$ blank screen. The total number of trials in this block was 30 .

\section{EEG Recording and Data Reductions}

The EEG was acquired with 128-channel HydroCel Geodesic sensor nets (Electrical Geodesics, Eugene, OR) and recorded/analysed with Net Station 4.3 software. Eye movements were recorded sensor net electrodes placed approximately $1 \mathrm{~cm}$ below the participants' right and left eyes.

For each subject's data the EEG was filtered with a .03 Hz high-pass and a $30 \mathrm{~Hz}$ lowpass filter. The EEG was then segmented for each trial from negative $200 \mathrm{~ms}$ to $1500 \mathrm{~ms}$ relative to the stimulus onset. Artefact detection included removing trials with amplitude changes above $150 \mu \mathrm{V}$ across an entire segment, as well as trials with eye blinks or lateral eye movements. Standard bad channel detection and replacement was then performed. The EEG epochs were then averaged to create the stimulus-locked ERPs for each subject. The singlesubject ERPs were then average referenced and baseline corrected (-200 to $0 \mathrm{~ms}$ ). For the neutral view condition, the mean number of trials was $24.88(\mathrm{SD}=4.27$, range $=14$ to 30$)$. For the negative view condition, the mean number of trials was $23.5(\mathrm{SD}=4.46$, range $=14$ to 30$)$. For the expressive suppression condition, the mean number of trials was 26.9 ( $\mathrm{SD}=3.56$, range $=16$ to 30$)$ 
Based on previous studies (Moser et al., 2006; Hajcak \& Dennis, 2009; Dennis \& Hajcak, 2009), the LPP was defined as the mean amplitude in three time windows following the stimulus onset: early (350 to $600 \mathrm{~ms}$ ), middle (600 to $1000 \mathrm{~ms}$ ), and late (1000 to $1500 \mathrm{~ms}$ ) time windows. As per these previous studies, we defined an LPP as a positive amplitude that occurs 1) after stimulus onset, 2) relative to the start of the trial, 3) can be easily seen in topomaps at posterior sites, and 4) extended from about $~ 300 \mathrm{~ms}$ to the end of the trial. ERPs were computed from three channels along the midline: central parietal $(\mathrm{CPz})$, parietal $(\mathrm{Pz})$ and occipital $(\mathrm{Oz})$, the same channels used in previous studies (Moser et al., 2006; Hajcak \& Dennis, 2009) for analyses of LPP activity. While the choice of Oz is unusual based on adult studies (Moser et al., 2006), it was selected for this study due to the LPP-like effects reported in children at occipital electrodes (Hajcak \& Dennis, 2009).

\section{Results}

\section{ERP Results}

A 3 (Channel: CPz, Pz, Oz) x 3 (Time window: early [350-600 ms], middle [600-1000 ms], late [1000-1500 ms]) x 3 (Condition: neutral view, negative view, expressive suppression) repeated measures analysis of variance (ANOVA) (see Table $\mathbf{1}$ for means and standard deviations, and Table 2 for t-test statistics) identified significant main effects of: channel $\mathrm{F}(2$, $51)=31.4, \mathrm{p}<.001 ;$ time window $\mathrm{F}(2,51)=84.32, \mathrm{p}<.001 ;$ and condition $\mathrm{F}(2,51)=14.58$, $p<.001$. There were significant interactions of: condition and time window $F(4,49)=27.12, p$ $<.001$; condition and channel $\mathrm{F}(4,49)=22.15, \mathrm{p}<.001$; and condition, time window and channel $\mathrm{F}(8,45)=10.27, \mathrm{p}<.001$. Post-hoc comparisons were Bonferroni-corrected. The significant threshold was set to .05 . The above results confirm the impression gathered from Figure 1 and Figure 2 that the three experimental conditions clearly differ between each other 
across a majority of recording sites and time windows. Table 1 presents the results of the post hoc paired tests conducted for each condition, at all time windows, across all channels.

At $\mathrm{CPz}$ channel, expressive suppression enhanced the LPP in all three time windows compared to both neutral and negative view conditions. Further, the negative view amplitude at middle and late windows was higher than in the neutral view condition. Compared to the early time window, there were relative positive-going increases in amplitudes in all three conditions. The neutral view condition had only negative (below-zero) amplitude values across all time windows, the negative view condition only had positive (above-zero) amplitudes after the early time window, and only the expressive suppression condition had positive amplitude values throughout all time windows.

At the Pz channel, expressive suppression enhanced the LPP in the early time window compared to both neutral and negative view conditions. The mean amplitudes in the middle time window for expressive suppression condition was not significantly different from the negative view condition, and both were significantly higher than the neutral view condition amplitude. There were no differences in late window for LPP amplitudes between the neutral view and expressive suppression conditions, whereas the negative view condition led to significantly higher LPP amplitudes than the neutral view condition. Further, the negative view amplitude at the early time window was higher than in the neutral view condition. In addition, LPP amplitudes did not differ in the late time window between the negative view and the expressive suppression conditions.

At the $\mathrm{Oz}$ channel, expressive suppression reduced the LPP in all three time windows compared to both neutral and negative view conditions. The three conditions differed from one another in both early and middle time windows. The neutral view condition led to a lower amplitude than the negative view condition in these two time windows. In the late time window 
there was no difference in amplitudes between the neutral and negative view conditions, whereas the expressive suppression condition amplitude remained lower than the other two conditions.

\section{Relationship between LPP and Age and Gender}

In order to examine how the changes in LPP relate to age, Pearson correlations between the mean LPP amplitude and age were calculated for each condition and time window. The correlations between LPP amplitudes and gender were computed in the same manner. The pvalues were corrected via Bonferroni adjustment for family-wise error.

Significant negative correlations with age that passed a Bonferroni correction (18 tests, adjusted $\mathrm{p}=.002$ ) were only found for the expressive suppression condition across all time windows at the occipital $(\mathrm{Oz})$ channel: early window $(\mathrm{r}=-.44, \mathrm{p}<.001)$; middle window 600 $1000(r=-.45 p<.001)$; late window 1000-1500 $(r=-.42 p<.01)($ See Figure 3). There were no significant correlations between age and $\mathrm{CPz}$ amplitudes. There were several other negative correlations that were below the significance threshold following the Bonferroni correction, including negative correlations of age with negative view amplitudes at the Oz channel in the early $(\mathrm{r}=-.34, \mathrm{p}=.013)$, middle $(\mathrm{r}=-.38, \mathrm{p}=.005)$ and late $(\mathrm{r}=-.32, \mathrm{p}=.018)$ time windows. Further, age was negatively correlated with expressive suppression amplitudes at $\mathrm{Pz}$ channel in the early time window $(r=-.33, r=.015)$. There were no positive correlations approaching significance between age and LPP metrics. These results suggest that LPP decreased with increasing age.

Furthermore, Hotelling's t analysis showed that the correlations between age and the expressive suppression LPP at the early time window were significantly higher compared to those with the negative view LPP, $\mathrm{t}=1.9, \mathrm{p}<.05$. This confirms the hypothesis that the amplitude decrease associated with age was related to the expressive suppression condition and 
not just the valence of the stimuli. In exploratory analyses we examined and found no significant point biserial correlations of gender with LPP values.

\section{Discussion}

The aim of the present study was to extend previous findings regarding the neural correlates of emotion regulation in early to late adolescence. This study provides new evidence that expressive suppression can alter brain activity associated with emotional responses to unpleasant stimuli in adolescents.

The present findings confirmed that, in line with previous studies (Cuthbert et al., 2000; Dillon, Cooper, Grent, Woldorff \& LaBar, 2006; Foti \& Hajcak, 2008; Moser et al., 2006), negative images increased the LPP compared to neutral images across all the examined channels. These results support the assumption that, as in findings from adult studies, the LPP is a valid metric to explore emotion regulation processes in adolescence. Further, we confirmed that expressive suppression in adolescents led to changes in the LPP, as has also been shown with adults and children with other emotion regulation strategies. This indicates that the LPP is sensitive to expressive suppression instructions and can be used to explore emotion regulation processes in adolescents.

Expressive suppression significantly reduced the LPP at occipital channels. Similarly to adult studies (Foti \& Hajcak, 2008), in the late time window, negative images preceded by a suppression cue resulted in parietal LPPs that were not significantly different to those elicited by neutral images. The negative view condition only differed from the neutral view condition, whereas the expressive suppression condition did not differ from the neutral view. Overall these findings suggest that, similarly to adults, adolescents can successfully use emotion regulation strategies to down-regulate emotional arousal. However, our results showing differences between passive viewing and expressive suppression conditions, should be 
interpreted with caution due to the current study design which did not allow for counterbalancing, and in particular due to the possible confounding effects of always having the passive viewing conditions before the expressive suppression condition (i.e., condition order).

Based on visual examination of Figure 2, at early time points in the expressive suppression condition (between 100-200 ms) there seems to be an amplitude decrease at $\mathrm{Oz}$, and increased amplitudes at $\mathrm{CPz}$ and Pz. This early modulation of the ERP suggests that the specific protocol used in this study, which included a suppression cue before the onset of an image altered the neural activity soon after the start of each trial. This trend may reflect the deployment of suppression strategies from the onset of the cue and prior to the onset of the image.

While adult studies do not usually report on changes at the occipital channel, in the present study the LPP reduction during expressive suppression in the late window was pronounced, such that the LPP was lower than in the neutral view condition, possibly this late window modulation of the LPP is indexing a later cognitive process beyond the mere diminution of earlier perceptual and emotional responses. Consistent with this idea, in the late time window, at the Oz channel, there was no significant difference between the negative view and neutral view conditions, whereas the effects of expressive suppression were evident through all the three time windows. The general suppression-induced LPP decrease at Oz may reflect a modulation of visual-sensory processing by emotional regulation. It is possible that this trend is more evident in the adolescent population, as previous studies (Hajcak \& Dennis, 2009; Stephanou et al., 2016; Wessing et al., 2015) have shown that the neural correlates of emotion regulation tend to be located in more occipital and posterior regions in younger participants. 
At the central-parietal $(\mathrm{CPz})$ channel the negative view condition did not generate an LPP (above-zero amplitude) in the early time window, but generated an LPP in the two late time windows. The expressive suppression condition resulted in an LPP in all three time windows, which was consistently higher than the negative and neutral view LPPs. The overall amplitude trend in CPz channel is similar to the one reported by Moser et al. (2006). It may be the LPP dynamics at CPz more closely reflect the attentional-cognitive demands of expressive suppression as instantiated by dorsal-parietal networks, in contrast to the more visualperceptual processes that are likely reflected by LPP dynamics at occipital channels, although this idea warrants further investigation. Increases in early LPP windows for both $\mathrm{Pz}$ and $\mathrm{CPz}$ may both reflect an increased effort deployed during expressive suppression. It is possible that the pre-emptive nature of the expressive suppression protocol (with suppression cue before image onset) used in this study led to considerably more effort from the start of the trial.

The scalp topography changes noted in this study, particularly the reduced occipital activity during expressive suppression, might be related to attenuated amygdala and visual cortex activation during emotion suppression tasks, as shown in multiple previous studies with adults and adolescents (Hayes et al., 2010; Hennenlotter et al., 2009; Stephanou et al., 2016; Wessing et al., 2015). However, this pattern of activity requires future interpretation in relation to the broader set of brain networks involved in both emotion generation and regulation. For example, although there have have been very few fMRI studies of expressive suppression, meta-analytic reviews suggest that expressive suppression, relative to reappraisal and other strategies, engages inferior frontal gyrus and temporal-parietal junction regions (Morawetz et al., 2016). Thus expressive suppression may have some unique neural features relative to other emotion regulation strategies that are likely to involve more cognitive-semantic processes..

As far as the temporal features of emotion regulation are concerned, the findings of the present study seem to confirm that some forms of response modulation, specifically expressive 
suppression, need not always be deployed after the emotion is generated but may be deployed early as a preventative regulatory strategy. This is consistent with the findings of Paul and colleagues (2013) and Vanderhasselt and colleagues (2013), suggesting that preparatory or "preventative" expressive suppression can be used when the content of the stimulus cannot be anticipated and an effective reappraisal is not immediately possible.

Consistent with prior studies that indicated general age-related decreases of visual ERP amplitudes (Taylor et al., 2004; Kuefner et al., 2010; Sumich et al., 2012) and LPP-related occipital source activity (Wessing et al., 2015), the general pattern of the present study was also of overall decreasing ERP amplitudes, especially at occipital channels. Moreover, as predicted, the current study found evidence of developmental differences in the neural correlates of emotion-regulation. Specifically, we found negative associations between the LPP reductions in the emotion regulation condition and age across early to late adolescence (from $12-17$ years). Overall the association with age is consistent with previous findings that reported neural correlates of emotion regulation change across development (McRae et al., 2012). More specifically, the results are consistent with findings from Kisley and colleagues (2007) showing that LPP to unpleasant images is reduced across the lifespan. The current findings suggest that this age-LPP association in part reflects a process of improved emotion regulation with maturation. This may indicate that the capacity to regulate becomes less effortful during the age span covered by this study, primarily due to normative psychological and neural maturation. In line with evidence that adults regulate emotions more efficiently than children (Pfeifer \& Blakemore, 2012), our findings suggest that older adolescents, relative to younger adolescents, also have a greater capacity to regulate their emotions. However, because there was no direct measurement of subjective emotional changes, it is not possible to address the effectiveness of our protocol in reducing negative emotion felt by the participants. 
Emotion regulation is a complex process with multiple components (Gross, 2015). Dan-Glauser and Gross (2011) describe an anticipatory autonomic/respiratory activity associated with expressive suppression in adults that occurs at the same time window ( 0 to 0.5 seconds) as the early parietal LPP increase during the expressive suppression condition in the present study. It is possible that this LPP increase is an EEG marker of the same preparatory "physiological suppression" process reported by Dan-Glauser and Gross (2011). While adults use emotion regulation strategies based on experience (Zimmerman \& Iwanski, 2014) and with relatively low effort, adolescents may be less prepared, in terms of learning and maturation, for the variety of emotionally evocative situations with which they are faced. Therefore the anticipatory activity involved with such physiological suppression is likely to demand more effort in adolescents compared to adults.

Zimmerman and Iwanski (2014) also showed that emotion regulation develops in an emotion specific manner, demonstrating an increased use of expressive suppression for fear from early adolescence to adulthood, but not for other emotions such as anger and sadness. Due to the nature of the IAPS images, which consist of some images that evoke fear, it is possible that the LPP decrease associated with age is linked to more habitual use of expressive suppression with increasing age in the current sample. It could be argued that the use of expressive suppression to deal with fear is adaptive, and the ability to use it improves with age. For example, concealing one's one fear may help in situations of danger (e.g., not showing fear to a threatening individual), whereas in other circumstances it may be part of acceptable social display rules (e.g., not showing personal fears in a group). However, the developmental pruning of personal emotion regulation strategies occurs in an idiosyncratic and context-dependent manner that is not yet well understood. Further, an additional possible explanation for the age 
related LPP reductions in both expressive suppression and negative view conditions may involve greater familiarity with extreme graphic media in the older adolescents.

More generally, it is interesting to consider the possible effects that may be unique to expressive suppression as a pre-emptive regulation strategy in adolescents as compared to antecedent-focused and response-modulation strategies. An individual may need to regulate the expression of emotions for a multitude of reasons such as: to comply to the social norms of a situation and adhere to display rules (not laughing at the teacher); to avoid others knowing what one actually feels (not showing a bully you are afraid); to help someone else to regulate his/her emotions (not showing one's anxiety to a younger sibling in a potentially dangerous situation). Broadly speaking, it is likely that the degree of adaptive expression of one's emotions varies greatly across different situations, relationships and cultures (Cole, Bruschi \& Tamang, 2002; English et al., 2016; Yeh, Bedford, Wu, Wang \& Yen, 2017).

Given the importance of peer relations in adolescence (Collins \& Laursen, 2004), one's expressive behavior may often be under significant social scrutiny by peers, hence not showing one's emotions may often be an adaptive behavior. As adult studies have found that social context is relevant to expressive suppression (English et al., 2016) and enhancement (Burton \& Bonanno, 2016), it is possible that the association between expressive suppression and age in the current study is driven by the fact that younger adolescents are less experienced in hiding their expressive behaviors as they are relatively "new" to the peer-focused context, while older adolescents may be significantly more practiced in it. This idea of practice and maturation effects due to social context is also in line with the hypothesis that suppression of fear may become more adaptive with increasing age, autonomy and responsibility (Zimmerman \& Iwanski, 2014). One might also suggest that younger adolescents' relative lack of experience means there are more situations and emotions which would be novel and highly arousing to them. Hence pre-emptive, context-independent and generalized emotion regulation strategies 
would be used more frequently and generally be more beneficial for this age group. Hence suppression can operate by targeting emotional responses early, prior to a fully expressed emotional response. Adolescents may frequently use expressive suppression as it seems to be an effective strategy of emotion regulation when faced with uncertainty. The findings of the present study seem to support the view that expressive suppression in adolescents can successfully modify emotions relatively early during emotion generation and regulation processes.

\section{Limitations and Possibilities for Future Research}

Several limitations should be considered when interpreting the current findings. First, the images we used were not classified according to specific emotion categories (e.g., fear, disgust), but into broad neutral and negative emotional categories. Therefore, it is difficult to reach conclusions about any emotion-specificity in the effects of expressive suppression on the LPP. This is a significant limitation as emotion regulation may develop in an emotion-specific manner (Zimmerman \& Iwanski, 2014). Second, we did not collect self-report emotion ratings and were not able to assess the effectiveness of expressive suppression in reducing the intensity of negative emotions felt. Third, the images were somewhat smaller than in past paradigms, thus the total emotional impact of the images on participants may have been somewhat diminished. Fourth, we did not control for social anxiety that may have been brought about due to being watched on video by the researcher while suppressing. Fifth, only one emotion regulation strategy was explored as part of this study, not allowing for comparisons between various emotion strategies, or their differential associations with age. It is important that future studies explore and compare multiple regulation strategies across a range of emotions. Sixth, the analyses were limited by being focused on a few single channels, and by being crosssectional for age analyses. 
In line with previous studies mentioned in the methods section, this study did not use a counterbalanced experimental design in order to avoid potential carryover effects of doing expressive suppression before the passive view conditions, thus likely maximizing the suppression challenge. However this experimental design results in a frequently reported tradeoff of not being able to be sure that the study effects were due to the possible differences between conditions and, specifically, their sequential effects on each other. The effects we have reported could be driven by or confounded with several factors including condition order, priming, habituation, cognitive load, and fatigue; and not due to the expressive suppression instructions per se. We attempted to minimize possible habituation effects between the passive viewing and expressive suppression blocks by using unique negative stimuli of similar intensity across the two conditions. Relatedly, Murata and colleagues (2012) pointed out that habituation effects should be minimal due to the robust LPP reactivity with repeated presentations of IAPS images (see Olofsson \& Polich, 2007 for review). Further, the two conditions differed in the way the instructions were presented, thus the reported study effects in the expressive suppression condition relative to passive viewing conditions may have also been influenced by the pre-trial audiovisual cue. Overall, while our findings should be taken with caution until replication and extension, the general pattern of ERP findings reported here matches well with published results using similar emotion regulation instructions. Thus it is our view that the main ERP effects related to expressive suppression were at least partly influenced by the expressive suppression instructions. In future studies the limitations related to counterbalancing could be addressed through arranging two separate visits for the participants as well as introducing ratings of stimulus intensity and cognitive effort.

As the parameters of expressive suppression tasks are not well studied, particularly with EEG and pediatric samples, the present study was designed to encourage an explicit use of expressive suppression and reminded the participant to use this regulation technique before 
each trial. However this reminder was delivered in a form of an audio-visual cue which was not present in the passive viewing conditions, therefore it is possible that the results of the study were partially influenced by this difference between the two conditions. We believe that if there are confounding effects from pre-trial cues, they most likely influenced early aspects of the trials and not the main LPP effects. Further, the pre-trial cue was early enough before the onset of the stimulus $(2500 \mathrm{~ms})$ to probably minimize pre-trial cue effects on the ERPs in this study.

An additional last limitation is related to the age range of participants in this study. We focused on the age range between 12 and 17; however, there is evidence of brain development associated with emotion regulation continues well into the 20 's and may be modified throughout the lifespan (Blakemore \& Choudhury, 2006). In order to further our understanding of change in emotion regulation associated with development, future studies should explore the neural correlates of emotion regulation strategies across broader age spans, or indeed, the entire life span.

\section{Conclusion}

The present study is the first to examine the ERP correlates of expressive suppression in an adolescent sample. This study had successfully replicated and extended previous adult findings by demonstrating that the LPP is sensitive to emotion regulation instructions in an adolescent population. The main findings were 1) age-related LPP decreases suggestive of greater facility with expressive suppression in older adolescents, which is likely due to normative developmental changes in emotion regulation networks; 2) an occipitally-focused LPP, not reported in adults but previously found in child studies, and 3) early, middle, and late window LPP effects due to expressive suppression. Further, both LPP increases and decreases were noted, depending on electrode site and time window. More broadly speaking, the current 
findings support the idea that expressive suppression is effective in significantly modulating the neural correlates of emotion regulation in adolescents. The decreased LPP with increasing age in our study may serve as a useful metric of normative brain and emotion regulation maturation. With continued exploration of the experimental constraints on the LPP and individual-difference variations in the LPP, it may come to serve as clinically relevant marker to index emotion-regulation processes in normally developing adolescents and those at risk for psychopathology. 
Table 1. Mean values and standard deviations (SD) and corresponding differences in post-hoc paired tests, between Neutral View, Negative view, and Expressive suppression trials, for every time epoch at each channel.

\begin{tabular}{|c|c|c|c|c|}
\hline $\begin{array}{c}\text { Time } \\
\text { Window }\end{array}$ & Channel & Neutral View & Negative View & $\begin{array}{c}\text { Expressive } \\
\text { Suppression }\end{array}$ \\
\hline \multirow{6}{*}{$\begin{array}{c}\text { Early } \\
\text { window }\end{array}$} & \multirow[t]{2}{*}{$\mathrm{CPz}$} & -4.51 & -3.82 & $3.16^{*}$ \\
\hline & & $(6.3 S D)$ & $(6.02 S D)$ & $(4.42 S D)$ \\
\hline & \multirow[t]{2}{*}{$\mathrm{Pz}$} & $5.14^{*}$ & $7.57^{*}$ & $10.96^{*}$ \\
\hline & & $(5.5 S D)$ & $(6.83 S D)$ & $(5.55 S D)$ \\
\hline & \multirow[t]{2}{*}{$\mathrm{Oz}$} & $14.84 *$ & $16.64^{*}$ & $12.72^{*}$ \\
\hline & & $(8.89 S D)$ & $(10.96 S D)$ & $(9.07 S D)$ \\
\hline \multirow{6}{*}{$\begin{array}{l}\text { Middle } \\
\text { window }\end{array}$} & \multirow[t]{2}{*}{$\mathrm{CPz}$} & $-0.78^{*}$ & $1.44^{*}$ & $4.92 *$ \\
\hline & & $(4.59 S D)$ & $(4.26 S D)$ & $(3.70 S D)$ \\
\hline & \multirow[t]{2}{*}{$\mathrm{Pz}$} & $3.24^{*}$ & 7.64 & 6.84 \\
\hline & & $(4.27 S D)$ & $(5.88 S D)$ & $(4.97 S D)$ \\
\hline & \multirow[t]{2}{*}{$\mathrm{Oz}$} & $8.49^{*}$ & $11.25^{*}$ & $5.57 *$ \\
\hline & & $(6.76 S D)$ & $(8.45 S D)$ & (6.11 SD) \\
\hline \multirow{6}{*}{$\begin{array}{c}\text { Late } \\
\text { Window }\end{array}$} & \multirow[t]{2}{*}{$\mathrm{CPz}$} & $-0.99 *$ & $1.07 *$ & $2.96^{*}$ \\
\hline & & $(4.41 S D)$ & $(3.66 S D)$ & $(3.58 S D)$ \\
\hline & \multirow[t]{2}{*}{$\mathrm{Pz}$} & $1.35^{*}$ & 3.41 & 2.06 \\
\hline & & $(3.56 S D)$ & $(4.25 S D)$ & $(3.80 S D)$ \\
\hline & \multirow[t]{2}{*}{$\mathrm{Oz}$} & 5.02 & 5.41 & $0.93 *$ \\
\hline & & $(5.58 S D)$ & $(6.43 S D)$ & $(4.52 S D)$ \\
\hline
\end{tabular}

Note: $*=$ condition is significantly different from both other conditions. 
Table 2. Post-hoc pairwise contrasts across time windows, channels, and conditions

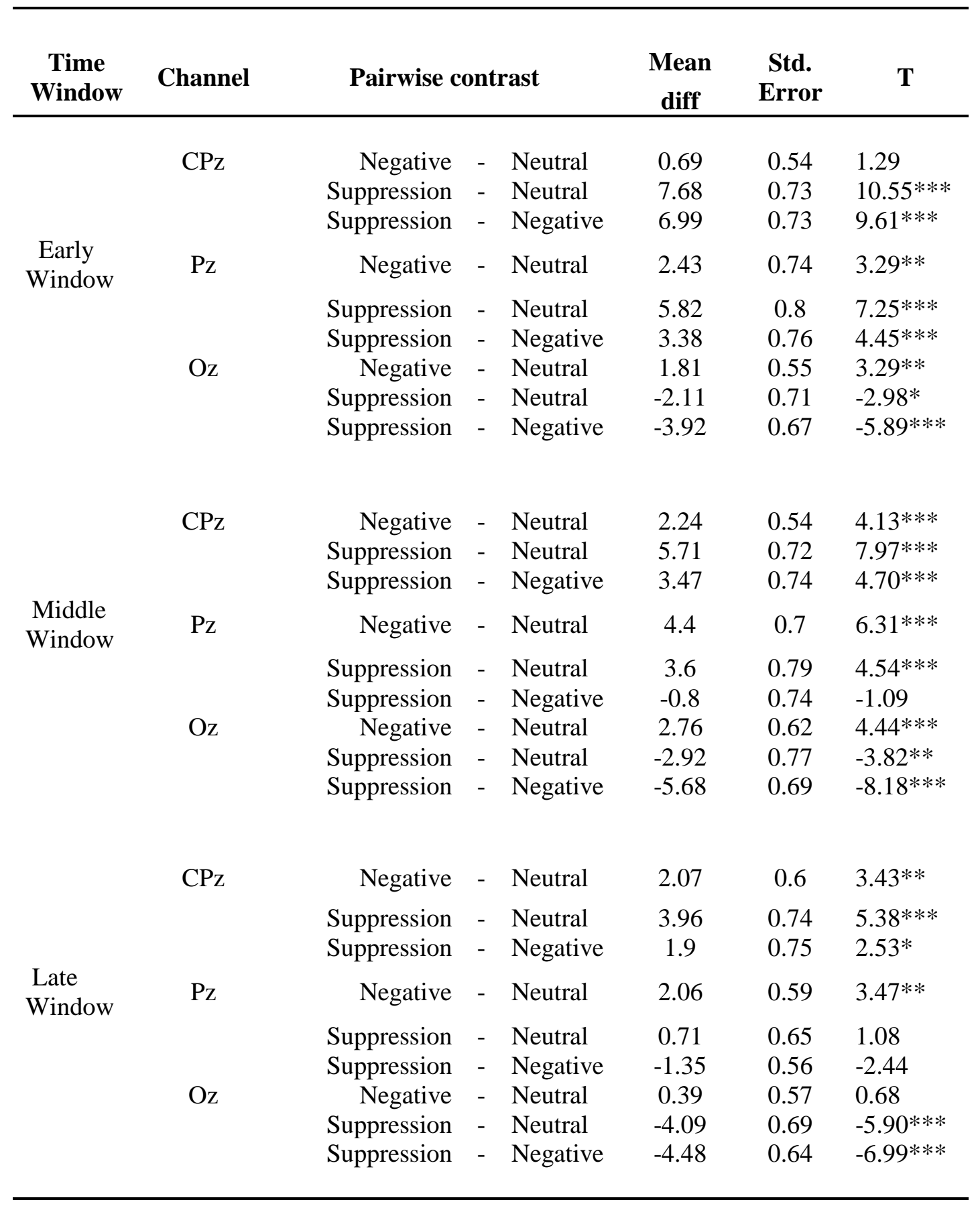

Note: Bonferroni corrected significance values. $*=<.05, * *=<.01$, $* * *=<.001$ 
Figure 1. Stimulus-locked ERPs at $\mathrm{CPz}, \mathrm{Pz}$ and $\mathrm{Oz}$ channels for Expressive Suppression, Negative View, and Neutral View conditions 
Figure 2. Scalp topographies for expressive suppression, negative view, and neutral view conditions at early, middle, and late LPP time windows. 
Figure 3. Scatterplot of correlations between $\mathrm{Oz}$ LPP and age. 


\section{References}

Adrian, M., Zeman, J., \& Veits, G. (2011). Methodological implications of the affect revolution: A 35-year review of emotion regulation assessment in children. Journal of experimental child psychology, 110(2), 171-197. http://dx.doi.org/10.1016/j.jecp.2011.03.009.

Aldao, A., Nolen-Hoeksema, S., \& Schweizer, S. (2010). Emotion-regulation strategies across psychopathology: A meta-analytic review. Clinical psychology review, 30(2), 217-237. http://dx.doi.org/10.1016/j.cpr.2009.11.004

American Psychiatric Association. (2013). Diagnostic and statistical manual of mental disorders (5th ed.). Arlington, VA: American Psychiatric Publishing.

Berking, M., Wirtz, C. M., Svaldi, J., \& Hofmann, S. G. (2014). Emotion regulation predicts symptoms of depression over five years. Behaviour research and therapy, 57, 13-20. http://dx.doi.org/10.1016/j.brat.2014.03.003

Blakemore, S. J., \& Choudhury, S. (2006). Development of the adolescent brain: implications for executive function and social cognition. Journal of child psychology and psychiatry, 47(3-4), 296-312. http://dx.doi.org/10.1111/j.1469-7610.2006.01611

Brenning, K., Soenens, B., Van Petegem, S., \& Vansteenkiste, M. (2015). Perceived maternal autonomy support and early adolescent emotion regulation: A longitudinal study. Social Development, 24(3), 561-578. http://dx.doi.org/ 10.1111/sode.12107

Burton, C. L., \& Bonanno, G. A. (2016). Measuring ability to enhance and suppress emotional expression: The Flexible Regulation of Emotional Expression (FREE) Scale. Psychological assessment, 28(8), 929. http://dx.doi.org/10.1037/pas0000231

Butler, E. A., \& Randall, A. K. (2013). Emotional coregulation in close relationships. Emotion Review, 5(2), 202-210. http://dx.doi.org/10.1177/1754073912451630 
Campbell-Sills, L., \& Barlow, D. H. (2007). Incorporating emotion regulation into conceptualizations and treatments of anxiety and mood disorders. In Gross, James J. (Ed). Handbook of emotion regulation, (pp. 542-559). New York, NY, US: Guilford Press.

Carstensen, L. L., Fung, H. H., \& Charles, S. T. (2003). Socioemotional selectivity theory and the regulation of emotion in the second half of life. Motivation and emotion, 27(2), 103-123. http://dx.doi.org/10.1023/A:1024569803230

Casey, B. J. (2015). Beyond simple models of self-control to circuit-based accounts of adolescent behavior. Annual Review of Psychology, 66, 295-319. http://dx.doi.org/10.1146/annurev-psych-010814-015156

Cole, P. M., Bruschi, C. J., \& Tamang, B. L. (2002). Cultural differences in children's emotional reactions to difficult situations. Child development, 73(3), 983-996. http://dx.doi.org/10.1111/1467-8624.00451

Collins, W. A., \& Laursen, B. (2004). Changing relationships, changing youth: Interpersonal contexts of adolescent development. The Journal of Early Adolescence, 24(1), 55-62.

Cuthbert, B. N., Schupp, H. T., Bradley, M. M., Birbaumer, N., \& Lang, P. J. (2000). Brain potentials in affective picture processing: covariation with autonomic arousal and affective report. Biological psychology, 52(2), 95-111. http://dx.doi.org/10.1016/S0301-0511(99)00044-7

Cutuli, D. (2014). Cognitive reappraisal and expressive suppression strategies role in the emotion regulation: an overview on their modulatory effects and neural correlates. Frontiers in Systems Neuroscience, 8. http://dx.doi.org/10.3389/fnsys.2014.00175

Dan-Glauser, E. S., \& Gross, J. J. (2011). The temporal dynamics of two response-focused forms of emotion regulation: Experiential, expressive, and autonomic consequences. 
Psychophysiology, 48(9), 1309-1322. http://dx.doi.org/10.1111/j.1469-

8986.2011.01191.x

de Rover, M., Brown, S. B., Boot, N., Hajcak, G., van Noorden, M. S., van der Wee, N. J., \& Nieuwenhuis, S. (2012). Beta receptor-mediated modulation of the late positive potential in humans. Psychopharmacology, 219(4), 971-979. http://dx.doi.org/10.1007/s00213-011-2426-x

DeCicco, J. M., Solomon, B., \& Dennis, T. A. (2012). Neural correlates of cognitive reappraisal in children: an ERP study. Developmental cognitive neuroscience, 2(1), 70-80. http://dx.doi.org/10.1016/j.den.2011.04.002

Dennis, T. A., \& Hajcak, G. (2009). The late positive potential: a neurophysiological marker for emotion regulation in children. Journal of Child Psychology and Psychiatry, 50(11), 1373-1383. http://dx.doi.org/10.1111/j.1469-7610.2009.02168.x

Dillon, D. G., Cooper, J. J., Grent, T., Woldorff, M. G., \& LaBar, K. S. (2006). Dissociation of event-related potentials indexing arousal and semantic cohesion during emotional word encoding. Brain and cognition, 62(1), 43-57.

http://dx.doi.org/10.1016/j.bandc.2006.03.008

English, T., Lee, I. A., John, O. P., \& Gross, J. J. (2016). Emotion regulation strategy selection in daily life: The role of social context and goals. Motivation and Emotion, $1-13$.

Esbjørn, B. H., Bender, P. K., Reinholdt-Dunne, M. L., Munck, L. A., \& Ollendick, T. H. (2012). The development of anxiety disorders: considering the contributions of attachment and emotion regulation. Clinical child and family psychology review, 15(2), 129-143. http://dx.doi.org/10.1007/s10567-011-0105-4 
Fernandez, K. C., Jazaieri, H., \& Gross, J. J. (2016). Emotion Regulation: A Transdiagnostic Perspective on a New RDoC Domain. Cognitive Therapy and Research, 40(3), 426440. http://dx.doi.org/10.1007/s10608-016-9772-2

Foti, D., \& Hajcak, G. (2008). Deconstructing reappraisal: Descriptions preceding arousing pictures modulate the subsequent neural response. Journal of Cognitive Neuroscience, 20(6), 977-988. http://dx.doi.org/10.1162/jocn.2008.20066

Fuhrmann, D., Knoll, L. J., \& Blakemore, S. J. (2015). Adolescence as a sensitive period of brain development. Trends in cognitive sciences, 19(10), 558-566. http://dx.doi.org/10.1016/j.tics.2015.07.008

Garnefski, N., Kraaij, V., \& van Etten, M. (2005). Specificity of relations between adolescents' cognitive emotion regulation strategies and internalizing and externalizing psychopathology. Journal of Adolescence, 28(5), 619-631. http://dx.doi.org/10.1016/j.adolescence.2004.12.009

Giordano, P. C. (2003). Relationships in adolescence. Annual review of Sociology, 29(1), 257-281. http://dx.doi.org /10.1146/annurev.soc.29.010202.100047

Gresham, D., \& Gullone, E. (2012). Emotion regulation strategy use in children and adolescents: The explanatory roles of personality and attachment. Personality and Individual Differences, 52(5), 616-621. http://dx.doi.org/10.1016/j.paid.2011.12.016

Gross, J. J. (1998a). Antecedent-and response-focused emotion regulation: divergent consequences for experience, expression, and physiology. Journal of personality and social psychology, 74(1), 224. http://dx.doi.org/10.1037/0022-3514.74.1.224

Gross, J. J. (1998b). The emerging field of emotion regulation: an integrative review. Review of general psychology, 2(3), 271. http://dx.doi.org/10.1037/1089-2680.2.3.271

Gross, J. J. (2015). Emotion regulation: Current status and future prospects. Psychological Inquiry, 26(1), 1-26. http://dx.doi.org/10.1080/1047840X.2014.940781 
Gross, J. J., \& Feldman Barrett, L. (2011). Emotion generation and emotion regulation: One or two depends on your point of view. Emotion review, 3(1), 8-16.

Gross, J. J., \& Levenson, R. W. (1993). Emotional suppression: physiology, self-report, and expressive behavior. Journal of personality and social psychology, 64(6), 970. http://dx.doi.org/10.1037/0022-3514.64.6.970

Gullone, E., Hughes, E. K., King, N. J., \& Tonge, B. (2010). The normative development of emotion regulation strategy use in children and adolescents: A 2-year follow-up study. Journal of Child Psychology and Psychiatry, 51(5), 567-574. http://dx.doi.org/ 10.1111/j.1469-7610.2009.02183.x

Hajcak, G., \& Dennis, T. A. (2009). Brain potentials during affective picture processing in children. Biological psychology, 80(3), 333-338. http://dx.doi.org/10.1016/j.biopsycho.2008.11.006

Hajcak, G., \& Nieuwenhuis, S. (2006). Reappraisal modulates the electrocortical response to unpleasant pictures. Cognitive, Affective, \& Behavioral Neuroscience, 6(4), 291-297. http://dx.doi.org/10.3758/CABN.6.4.291

Hajcak, G., MacNamara, A., \& Olvet, D. M. (2010). Event-related potentials, emotion, and emotion regulation: an integrative review. Developmental neuropsychology, 35(2), 129-155. http://dx.doi.org/10.1080/87565640903526504

Hayes, J. P., Morey, R. A., Petty, C. M., Seth, S., Smoski, M. J., McCarthy, G., \& LaBar, K. S. (2010). Staying cool when things get hot: emotion regulation modulates neural mechanisms of memory encoding. Frontiers in Human Neuroscience, 4, 230. http://dx.doi.org/10.3389/fnhum.2010.00230

Hennenlotter, A., Dresel, C., Castrop, F., Ceballos-Baumann, A. O., Wohlschläger, A. M., \& Haslinger, B. (2009). The link between facial feedback and neural activity within central circuitries of emotion-New insights from Botulinum toxin-induced 
denervation of frown muscles. Cerebral Cortex, 19(3), 537-542.

http://dx.doi.org/10.1093/cercor/bhn104

Kadosh, K. C., Luo, Q., de Burca, C., Sokunbi, M. O., Feng, J., Linden, D. E., \& Lau, J. Y. (2016). Using real-time fMRI to influence effective connectivity in the developing emotion regulation network. NeuroImage, 125, 616-626.

http://dx.doi.org/10.1016/j.neuroimage.2015.09.070

Keil, A., Moratti, S., \& Stolarova, M. (2003). Sensory amplification for affectively arousing stimuli: Electrocortical evidence across multiple channels. In Journal of Psychophysiology 17(3), 158-159.

Kim-Spoon, J., Cicchetti, D., \& Rogosch, F. A. (2013). A longitudinal study of emotion regulation, emotion lability-negativity, and internalizing symptomatology in maltreated and nonmaltreated children. Child development, 84(2), 512-527. http://dx.doi.org/10.1111/j.1467-8624.2012.01857.x

Kisley, M. A., Wood, S., \& Burrows, C. L. (2007). Looking at the Sunny Side of Life AgeRelated Change in an Event-Related Potential Measure of the Negativity Bias. Psychological Science, 18(9), 838-843. http://dx.doi.org/10.1111/j.1467$\underline{9280.2007 .01988 . x}$

Krompinger, J. W., Moser, J. S., \& Simons, R. F. (2008). Modulations of the electrophysiological response to pleasant stimuli by cognitive reappraisal. Emotion, 8(1), 132-137. http://dx.doi.org/10.1037/1528-3542.8.1.132

Kujawa, A., Hajcak, G., Danzig, A. P., Black, S. R., Bromet, E. J., Carlson, G. A., ... \& Klein, D. N. (2015). Neural reactivity to emotional stimuli prospectively predicts the impact of a natural disaster on psychiatric symptoms in children. Biological psychiatry. 80(5), 381-389. http://dx.doi.org/10.1016/j.biopsych.2015.09.008 
Kujawa, A., Klein, D. N., \& Proudfit, G. H. (2013). Two-year stability of the late positive potential across middle childhood and adolescence. Biological psychology, 94(2), 290-296. http://dx.doi.org/10.1016/j.biopsycho.2013.07.002

Kujawa, A., Klein, D. N., \& Hajcak, G. (2012). Electrocortical reactivity to emotional images and faces in middle childhood to early adolescence. Developmental cognitive neuroscience, 2(4), 458-467. http://dx.doi.org/10.1016/j.dcn.2012.03.005

Lang, P. J., Bradley, M. M., \& Cuthbert, B. N. (2008). International affective picture system (IAPS): Affective ratings of pictures and instruction manual. Technical report A-8.

Lewis, M. D., Lamm, C., Segalowitz, S. J., Stieben, J., \& Zelazo, P. D. (2006). Neurophysiological correlates of emotion regulation in children and adolescents. Journal of cognitive neuroscience, 18(3), 430-443. http://dx.doi.org/10.1162/jocn.2006.18.3.430

McRae, K. (2016). Cognitive emotion regulation: a review of theory and scientific findings. Current Opinion in Behavioral Sciences, 10, 119-124. http://dx.doi.org/10.1111/j.1467-8721.2008.00566.x

McRae, K., Gross, J. J., Weber, J., Robertson, E. R., Sokol-Hessner, P., Ray, R. D., ... \& Ochsner, K. N. (2012). The development of emotion regulation: an fMRI study of cognitive reappraisal in children, adolescents and young adults. Social cognitive and affective neuroscience, 7(1), 11-22. http://dx.doi.org/10.1093/scan/nsr093

Morawetz, C., Bode, S., Derntl, B., \& Heekeren, H. R. (2016). The effect of strategies, goals and stimulus material on the neural mechanisms of emotion regulation: A metaanalysis of fMRI studies. Neuroscience \& Biobehavioral Reviews. http://dx.doi.org/10.1016/j.neubiorev.2016.11.014 
Moser, J. S., Hajcak, G., Bukay, E., \& Simons, R. F. (2006). Intentional modulation of emotional responding to unpleasant pictures: an ERP study. Psychophysiology, 43(3), 292-296. http://dx.doi.org/10.1111/j.1469-8986.2006.00402.x

Murata, A., Moser, J. S., \& Kitayama, S. (2012). Culture shapes electrocortical responses during emotion suppression. Social Cognitive and Affective Neuroscience, nss036. https://doi.org/10.1093/scan/nss036

Musser, E. D., Backs, R. W., Schmitt, C. F., Ablow, J. C., Measelle, J. R., \& Nigg, J. T. (2011). Emotion regulation via the autonomic nervous system in children with attention-deficit/hyperactivity disorder (ADHD). Journal of abnormal child psychology, 39(6), 841-852. http://dx.doi.org/10.1007/s10802-011-9499-1

Nelson, B. D., Perlman, G., Hajcak, G., Klein, D. N., \& Kotov, R. (2015). Familial risk for distress and fear disorders and emotional reactivity in adolescence: An event-related potential investigation. Psychological medicine, 45(12), 2545-2556. http://dx.doi.org/10.1017/S0033291715000471

Olofsson, J. K., Nordin, S., Sequeira, H., \& Polich, J. (2008). Affective picture processing: an integrative review of ERP findings. Biological psychology, 77(3), 247-265. http://dx.doi.org/10.1016/j.biopsycho.2007.11.006

Paul, S., Kathmann, N., \& Riesel, A. (2016). The costs of distraction: The effect of distraction during repeated picture processing on the LPP. Biological psychology, 117, 225-234. http://dx.doi.org/10.1016/j.biopsycho.2016.04.002

Paul, S., Simon, D., Kniesche, R., Kathmann, N., \& Endrass, T. (2013). Timing effects of antecedent-and response-focused emotion regulation strategies. Biological psychology, 94(1), 136-142. http://dx.doi.org/10.1016/j.biopsycho.2013.05.019

Penela, E. C., Walker, O. L., Degnan, K. A., Fox, N. A., \& Henderson, H. A. (2015). Early behavioral inhibition and emotion regulation: pathways toward social competence in 
middle childhood. Child development, 86(4), 1227-1240.

http://dx.doi.org/10.1111/cdev.12384

Perlman, S. B., \& Pelphrey, K. A. (2011). Developing connections for affective regulation: age-related changes in emotional brain connectivity. Journal of experimental child psychology, 108(3), 607-620. http://dx.doi.org/10.1016/j.jecp.2010.08.006

Pfeifer, J. H., \& Blakemore, S. J. (2012). Adolescent social cognitive and affective neuroscience: past, present, and future. Social cognitive and affective neuroscience, 7(1), 1-10. http://dx.doi.org/10.1093/scan/nsr099

Pincham, H. L., Bryce, D., \& Pasco Fearon, R. M. (2015). The neural correlates of emotion processing in juvenile offenders. Developmental science, 18(6), 994-1005. http://dx.doi.org/10.1111/desc.12262

Schupp, H. T., Flaisch, T., Stockburger, J., \& Junghöfer, M. (2006). Emotion and attention: event-related brain potential studies. Progress in brain research, 156, 31-51. http://dx.doi.org/10.1016/S0079-6123(06)56002-9

Sheppes, G., Suri, G., \& Gross, J. J. (2015). Emotion regulation and psychopathology. Annual Review of Clinical Psychology, 11, 379-405. http://dx.doi.org/10.1146/annurev-clinpsy-032814-112739

Shulman, E. P., Harden, K. P., Chein, J. M., \& Steinberg, L. (2014). The Development of Impulse Control and Sensation-Seeking in Adolescence: Independent or Interdependent Processes?. Journal of Research on Adolescence. http://dx.doi.org/10.1111/jora.12181

Silk, J. S., Steinberg, L., \& Morris, A. S. (2003). Adolescents' emotion regulation in daily life: Links to depressive symptoms and problem behavior. Child development, 74(6), 1869-1880. http://dx.doi.org/10.1046/j.1467-8624.2003.00643.x 
Silvers, J. A., Insel, C., Powers, A., Franz, P., Helion, C., Martin, R. E., ... \& Ochsner, K. N. (2016). vlPFC-vmPFC-Amygdala Interactions Underlie Age-Related Differences in Cognitive Regulation of Emotion. Cerebral Cortex, bhw073. https://doi.org/10.1093/cercor/bhw073

Silvers, J. A., McRae, K., Gabrieli, J. D., Gross, J. J., Remy, K. A., \& Ochsner, K. N. (2012). Age-related differences in emotional reactivity, regulation, and rejection sensitivity in adolescence. Emotion, 12(6), 1235. http://dx.doi.org/10.1037/a0028297

Stephanou, K., Davey, C. G., Kerestes, R., Whittle, S., Pujol, J., Yücel, M., ... \& Harrison, B. J. (2016). Brain functional correlates of emotion regulation across adolescence and young adulthood. Human brain mapping, 37(1), 7-19. http://dx.doi.org/10.1002/hbm.22905

Thiruchselvam, R., Blechert, J., Sheppes, G., Rydstrom, A., \& Gross, J. J. (2011). The temporal dynamics of emotion regulation: an EEG study of distraction and reappraisal. Biological psychology, 87(1), 84-92. http://dx.doi.org/10.1016/j.biopsycho.2011.02.009

Thompson, R.A., Lewis, M.D., \& Calkins, S.D. (2008). Reassessing emotion regulation. Child Development Perspectives., 2(3), 124-131. http://dx.doi.org/10.1111/j.17508606.2008.00054.x

Tortella-Feliu, M., Balle, M., \& Sesé, A. (2010). Relationships between negative affectivity, emotion regulation, anxiety, and depressive symptoms in adolescents as examined through structural equation modeling. Journal of Anxiety Disorders, 24(7), 686-693. http://dx.doi.org/ 10.1016/j.janxdis.2010.04.012.

Tottenham, N., Hare, T. A., \& Casey, B. J. (2011). Behavioral assessment of emotion discrimination, emotion regulation, and cognitive control in childhood, adolescence, 
and adulthood. Frontiers in psychology, 2, 39.

http://dx.doi.org/10.3389/fpsyg.2011.00039

Uusberg, A., Thiruchselvam, R., \& Gross, J. J. (2014). Using distraction to regulate emotion: Insights from EEG theta dynamics. International Journal of Psychophysiology, 91(3), 254-260. http://dx.doi.org/10.1016/j.ijpsycho.2014.01.006

Vanderhasselt, M. A., Baeken, C., Van Schuerbeek, P., Luypaert, R., \& De Raedt, R. (2013). Inter-individual differences in the habitual use of cognitive reappraisal and expressive suppression are associated with variations in prefrontal cognitive control for emotional information: an event related fMRI study. Biological psychology, 92(3), 433-439. http://dx.doi.org/10.1016/j.biopsycho.2012.03.005

Van Rheenen, T. E., \& Rossell, S. L. (2014). Objective and subjective psychosocial functioning in bipolar disorder: An investigation of the relative importance of neurocognition, social cognition and emotion regulation. Journal of affective disorders, 162, 134-141. http://dx.doi.org/10.1016/j.jad.2014.03.043

Vijayakumar, N., Whittle, S., Dennison, M., Yuecel, M., Simmons, J., \& Allen, N. B. (2014). Development of temperamental effortful control mediates the relationship between maturation of the prefrontal cortex and psychopathology during adolescence: A 4-year longitudinal study. Developmental cognitive neuroscience, 9, 30-43. http://dx.doi.org/10.1016/j.den.2013.12.002

Werner, K., \& Gross, J. J. (2010). Emotion regulation and psychopathology: A conceptual framework. In Kring, A. M., \& Sloan, D. M. (Eds.). Emotion regulation and psychopathology: A transdiagnostic approach to etiology and treatment. Guilford Press.

Wessing, I., Rehbein, M. A., Postert, C., Fürniss, T., \& Junghöfer, M. (2013). The neural basis of cognitive change: Reappraisal of emotional faces modulates neural source 
activity in a frontoparietal attention network. NeuroImage, 81, 15-25. http://dx.doi.org/10.1016/j.neuroimage.2013.04.117

Wessing, I., Rehbein, M. A., Romer, G., Achtergarde, S., Dobel, C., Zwitserlood, P., ... \& Junghöfer, M. (2015). Cognitive emotion regulation in children: Reappraisal of emotional faces modulates neural source activity in a frontoparietal network. Developmental cognitive neuroscience, 13, 1-10. http://dx.doi.org/10.1016/j.den.2015.01.012

Yeh, K. H., Bedford, O., Wu, C. W., Wang, S. Y., \& Yen, N. S. (2017). Suppression Benefits Boys in Taiwan: The Relation between Gender, Emotional Regulation Strategy, and Mental Health. Frontiers in Psychology, 8. . http://dx.doi.org/10.3389/fpsyg.2017.00135

Zeman, J., Cassano, M., Perry-Parrish, C., \& Stegall, S. (2006). Emotion regulation in children and adolescents. Journal of Developmental \& Behavioral Pediatrics, 27(2), $155-168$.

Zhang, W., Li, H., Chen, J., Chen, N., Liu, X., Wang, D., \& Shen, J. (2014). Posterior P1 and early frontal negativity reflect developmental changes in attentional distraction during adolescence. Brain and cognition, 87, 30-38. http://dx.doi.org/10.1016/j.bandc.2014.02.011

Zhang, W., Lu, J., Fang, H., Pan, X., Zhang, J., \& Wang, D. (2012). Late positive potentials in affective picture processing during adolescence. Neuroscience letters, 510(2), 8892. http://dx.doi.org/10.1016/j.neulet.2012.01.008

Zhang, W., Lu, J., Ni, Z., Liu, X., Wang, D., \& Shen, J. (2013). Harm avoidance in adolescents modulates late positive potentials during affective picture processing. International Journal of Developmental Neuroscience, 31(5), 297-302. http://dx.doi.org/10.1016/j.ijdevneu.2013.03.009 
Zimmerman, P., \& Iwanski, A. (2014). Emotion regulation from early adolescence to emerging adulthood and middle adulthood Age differences, gender differences, and emotion-specific developmental variations. International Journal of Behavioral Development, 38(2), 182-194. http://dx.doi.org/10.1177/0165025413515405 